\title{
Twisted Square Plate Method and Other Methods for Determining the Shear Stress-Strain Relation of Flat Sheet ${ }^{* 1}$
}

\author{
Walter Ramberg and James A. Miller
}

\begin{abstract}
A method is presented for determining the stress-strain relation in shear for isotropic flat sheet. The method requires measurements of deflection or of extreme fiber bending strain in the center portion of a twisted square plate. The octahedral stress derived from the stress-strain curve in shear for a twisted plate of aluminum alloy agreed within about 5 percent with the octahedral stress derived from tensile and compressive tests of the same material. The difference was much greater for specimens of mild steel with a definite yield point. Unfortunately, the method is difficult to perform on thin sheet because of the small size of the specimen. An examination of alternate methods indicates particular promise for the use of an annulus of constant thickness.
\end{abstract}

\section{Introduction}

There has long been an interest in the stress-strain relations in shear of materials in the form of sheet. The interest has become more pronounced as working stresses and strains have increased and as it has become apparent that the mechanical properties of thin sheet could not be deduced directly from mechanical tests of heavier sections. The most satisfactory method, perhaps the only really satisfactory one, for determining the stress-strain curve of thin material in shear requires the material to be in the form of tubing with circular section. As long as the tube material is isotropic, its stress-strain curve in shear can be computed conveniently from the torquetwist curve of the tube by following the procedure given in $[1] .^{2}$

There is no dearth of suggestions for methods of determining the stress-strain curve in shear of flat sheet. Figure 1 indicates 10 suggested methods for stressing thin sheet in shear that have come to the authors' attention. The first two methods deal with tubular specimens.

Method (a) is nothing more than the bending of a strip of the sheet into a tube of large diameter, which is then tested like the conventional long tube for which a satisfactory theory exists [1]. The tube would have to have a large diameter so that the strains introduced in bending the strip would be small compared to the strains to be measured. Difficulties might be experienced with deviations from the theoretical uniform shearing stress caused by variations in clamping forces along the edges of the specimen. Difficulties might be experienced also with the discontinuity at the joint and with the transmission of sufficient torque to obtain stresses in the plastic range.

Method (b) was suggested to one of the authors

*This paper is based on work sponsored by the National Advisory Committee for Aeronautics and the Mechanics Branch, Office of Naval Research, U. S. Department of the Navy

1 The major portion of this paper was presented before the First U. S. National Congress of Applied Mechanics in Chicago on June 11 to 16, 1951. It was published in the Proceedings of that Congress under the title "Determinatior of Stress-Strain Curve in Shear by Twisting Square Plate:

2 Figures in brackets indicate the literature referencesat the end of this paper. by J. M. Frankland of Chance Vought Aircraft. Four strips of the sheets are assembled into a tube of square section. The strips will be subjected to a uniform shearing stress by equal and opposite torques applied to the ends, if the torsional rigidity of the joints along the four corners is negligible compared with the torsional rigidity of the tube as a whole. It is probable that this corner effect is not negligible for tubes that are sufficiently thick-walled to transmit, without buckling, torques that extend the shearing stresses into the plastic range.

Several methods have been suggested for stressing a specimen of sheet in shear by forces acting in the plane of the sheet.

Method (c) is the well-known square-picture-frame method, in which a square specimen is held between four pairs of rigid clamping bars with pin joints at the corners. The specimen will be placed in a state of uniform shear by equal and opposite forces acting at opposite corners of the frame, provided that the sheet is perfectly flat, the clamping bars are infinitely rigid, the edge effect due to deviations from ideal clamping does not extend into the middle portion of the sheet, and the pin joints are frictionless.

Method (d) tries to get around the difficulties with the edge effect in method (c) by making the specimen long so that the shearing stress is practically uniform along the middle portion of the specimen. However, an end effect will remain because the shearing stress reduces to zero at the corners. This end effect may be evaluated by making tests of the same material in picture frames of different lengths. The procedure would be costly and time consuming.

Method (e) was used by Coker and Filon [2] and Bollenrath [3] to stress thin sheet in shear. The loads are applied symmetrically to subject each one of the two "bays" of the specimen to a state of stress in which the shearing stress along the center line of each bay varies from zero at the ends to a nearly constant value near the middle. As in method (d), the end effect may be eliminated by testing specimens of different lengths.

Method (f), suggested by W. R. Osgood, subjects a single strip to a state of stress which approximates 


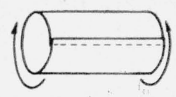

(a)

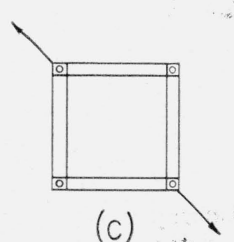

(c)

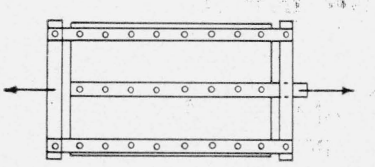

(e)

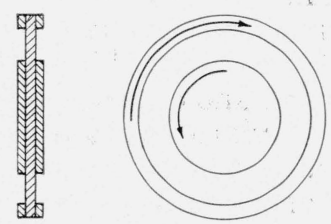

(g)

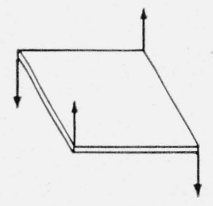

(i)

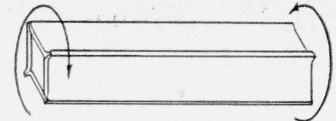

(b)

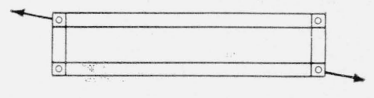

(d)

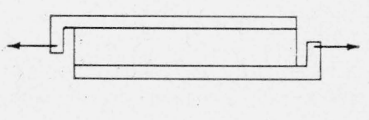

(f)

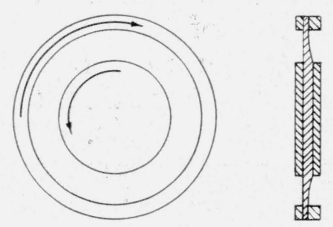

(h)

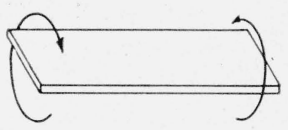

(j)
Figure 1. Methods suggested for stressing thin sheet in shear.

that in each bay of the specimen for method (e).

Method (g) avoids end effects by testing the sheet in the form of an "endless strip" or annulus. Equal and opposite torques are applied at the inner and outer edges of the annulus. The method has the advantage that, except near the periphery and the inner circle, the shearing stresses would be known accurately, for isotropic sheet, in the plastic as well as in the elastic range. There are some disadvantages, however. The shearing stress varies inversely as the square of the radius of the circle along which it acts. Hence there will be a nonlinear strain variation along the gage length of any finite gage mounted at $45^{\circ}$ to the tangential direction. As a result, the average strain along the gage will differ from the strain at the midpoint of the gage, unless the annulus is large enough to make the nonlinear variations negligible. If the annulus is large enough, it may be difficult to apply sufficient torque to stress the material into the plastic range. The testing machine would require heads that are rotatable about their axis only, to prevent bending of the specimen by rotation about other axes. The method would give only an average stress-strain curve for anisotropic sheet.
Method (h) eliminates some of the difficulties of method $(\mathrm{g})$. The annulus is machined into a disk that varies in thickness inversely as the square of the radius. The material in the annulus will be subjected in that case to a uniform shearing stress by equal and opposite torques applied at the inner and outer edge. The disk must be sufficiently thick near the periphery to develop strains in the plastic range before buckling occurs and the variation in thickness with radius must be small enough at the radius where the strains are measured to assure a practically uniform shearing stress throughout the thickness. A practical difficulty is that of machining the specimen accurately to the desired thickness variation without changing the properties of the material by the machining operation.

The methods so far mentioned subject the sheet to a shearing stress that is substantially uniform throughout the thickness of the sheet. In the remaining two methods the shearing stress is varied in the thickness direction from one extreme to an opposite extreme.

In method (i) a state of shear is produced in a square plate by twisting the plate into an anticlastic surface by equal and opposite tranverse forces applied to adjacent corners. The state of shear will be uniform for planes at a fixed distance from the median plane which will undergo zero shearing stress. The upper and lower faces of the plate will be under equal and opposite shearing stress. Objections might be raised against this method in so far as it neglects deviations from uniform shear due to various causes, such as having large transverse stresses near the corners and no shearing stresses along the edges. These objections will be discussed in detail in the body of the paper. Experimental difficulties may arise in applying the corner loads to the specimen without interfering with strain and deflection measurements on the faces of the plate.

Method (j), suggested by A. H. Stang, uses a much simpler scheme for producing a state of stress similar to that in method (i). A specimen in the shape of a long strip is placed in a state of shear by applying equal and opposite torques to the ends of the strin. Shearing strains can be measured with strain gages or they can be obtained from the twist per unit length of the strip. However, if the strip is made long enough to eliminate end effects, it is likely to deflect laterally. This will superimpose bending stresses on the torsional stresses. The same objections might be raised in this case as in case (i) with regard to edge effects. The shearing stress at the extreme fiber will be practically constant in the center portion, far from the edges of the strip, but it will decrease rapidly to zero as the edges are approached. The value of the extreme fiber shearing stress near the center of the strip will depend on this edge effect. It can be computed exactly in the elastic range, but no exact solution is known for the plastic range.

Consideration of the above 10 methods suggests that at least three of them, the square-picture-frame method (c), the annulus of constant thickness method 
$(\mathrm{g})$, and the twisted-square-plate method (i), should be tried out experimentally.

An experimental study of method (c), the square picture frame, was conducted by Wm. R. Osgood about 5 years ago. Method (c) was selected because it appeared to have particular promise and because it had been used in the aircraft industry to estimate shear properties of sheet. A fixture had been developed at the El Segundo Plant of the Douglas Aircraft Co. to make such tests. A duplicate of this fixture was obtained, and tests were made at the National Bureau of Standards for the National Advisory Committee for Aeronautics on several $4.5 \times 4.5 \times 0.063$ in. $24 \mathrm{~S}-\mathrm{T}$ aluminum alloy plates. The results were disappointing. 'They were reported to the NACA, but were not published. The strains were far from uniform even in the center portion of the plate farthest from the edges. The extreme fiber strains in this optimum region deviated up to 10 percent from the average strain. The deviations persisted even after an attempt had been made to improve the conditions of clamping along the edges by remachining parts of the clamping fixture.

Two years after completion of the work for the NACA, it was decided to try method (i), the twisted square plate, since Nadai had shown in 1915 [4] that this method provided a simple means for determining the shear modulus of sheet material. More recently the test has been extended to orthotropic materials at the Forest Products Laboratory [5] to determine the shear moduli of wood.

\section{Nomenclature}

The following nomenclature is used in the paper: $a=$ half-span for deflection measurements. $b=$ width of specimen .

$E=$ modulus of elasticity in tension or compression.

$G=$ modulus of elasticity in shear.

$h=$ thickness of specimen.

$m=$ moment per unit length.

$M=$ bending moment.

$M_{t}=$ twisting moment.

$P=$ load at each corner of plate.

$R=$ radius of curvature along diagonals of plate.

$u, v, w=$ displacements parallel to $x-, y$ - and $z$ axes, respectively.

$x, y=$ axes parallel to diagonals of plate, also planes normal to the corresponding axes.

$x^{\prime}, y^{\prime}=$ axes parallel to edges of plate, also planes normal to the corresponding axes.

$z=$ axis parallel to thickness of plate, also plane normal to $z$-axis.

$\gamma=$ shearing strain .

$\gamma_{o}=$ octahedral strain.

$\epsilon=$ normal strain.

$\nu=$ Poisson's ratio.

$\sigma=$ normal stress.

$\tau=$ shearing stress.

$\tau_{o}=$ octahedral stress.

\section{Principle}

\subsection{Elastic case}

An elastic square plate will be in a state of pure shear, in which the shearing stress is proportional to the distance from the neutral surface of the plate when the plate is bent into an anticlastic surface with equal and opposite principal curvatures. The bending of the plate into such an anticlastic surface produces equal and opposite extreme fiberbending stresses along the two principal axes. Nadai has shown that this remarkably simple state of stress may be realized by applying equal and opposite tranverse forces to adjacent corners of the plate.

Nadai's proof [4] makes use of Saint-Venant's principle of the equivalence of equipollent loads. Nadai notes that for a load $P$ at each corner, each edge of the plate is loaded by equal and opposite forces $P / 2$ acting at two corners (fig. 2). The load along the edge will remain unchanged upon applying balancing forces $\pm P / 2$ at frequent intervals along the edge, as indicated in fig. 2. Any element of length of edge $\Delta x^{\prime}$ between two adjacent equal and opposite forces $P / 2$ will then be subjected to a twisting moment $(P / 2) \Delta x^{\prime}$. According to Saint-Venant's principle, this edge load is equivalent to any edge load which results in a constant twisting moment per unit length

$$
m_{x^{\prime} y^{\prime}}=\frac{1}{\Delta x^{\prime}} \frac{P}{2} \Delta x^{\prime}=\frac{P}{2},
$$

uniformly distributed along the edge of the plate. The stress distribution, in regions of the plate sufficiently far from the edge to satisfy Saint-Venant's principle, will then be that corresponding to uniform twisting moments $+m_{x^{\prime} y^{\prime}},-m_{x^{\prime} y^{\prime}}$ in the $x^{\prime}, y^{\prime}$ planes, normal to the $x^{\prime}, y^{\prime}$ axes, respectively. The twisting moments $\pm m_{x^{\prime} y^{\prime}}$ are associated with principal bending moments $m_{x}, m_{y}$ on the $y, x$ planes normal to the diagonals of the plate. Equilibrium of moments acting on the edges of a triangular element of the plate (fig. 3) bounded by planes parallel to the $x, y$, and $y^{\prime}$ planes shows that

$$
m_{x}=m_{y}=-m_{x^{\prime} y^{\prime}}=-P / 2 .
$$

The plate is bent into an anticlastic surface by equal and opposite bending moments per unit length $P / 2$ acting on the $y, x$ planes normal to the diagonals of the plate. The surface will have its planes of prin-

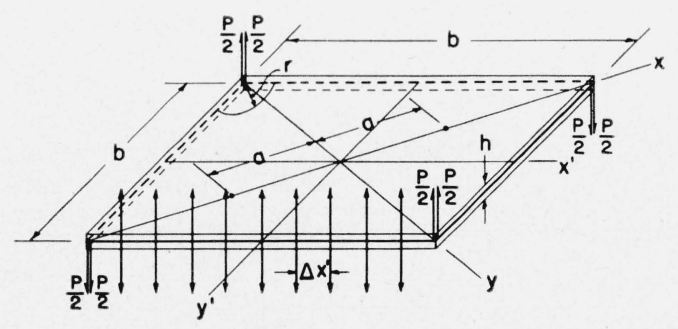

FiguRE 2. Twisting of square plate by forces on corners. 


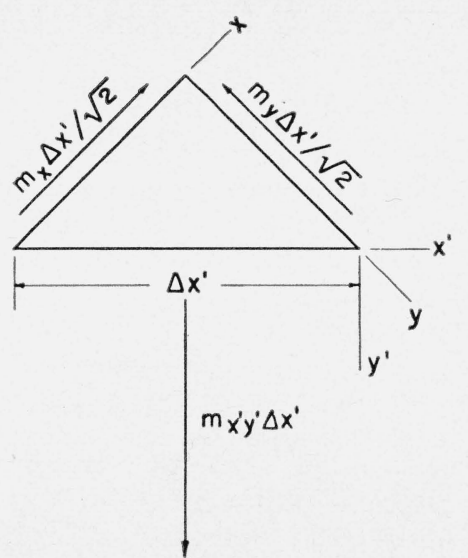

FIGURE 3. Edge moments acting on element of twisted square plate.

cipal curvature normal to the diagonals of the plate. If $R$ is the radius of curvature along the diagonals, the deflection relative to a plane tangent to the center of the plate is given by

$$
w=\frac{x^{2}-y^{2}}{2 R}
$$

The distribution of strain and of stress throughout the plate may be computed on the assumption of the Kirchhoff plate theory that plane sections remain plane. The normal strains $\epsilon_{x}, \epsilon_{y}$ must then increase linearly with distance from the neutral fiber

$$
\epsilon_{x}=\frac{2 z}{h} \epsilon_{1} \quad \epsilon_{y}=\frac{2 z}{h} \epsilon_{2},
$$

where $\epsilon_{1}, \epsilon_{2}$ are the principal extreme fiber strains; $z$, the distance from neutral fiber; and $h$, the thickness of plate. The maximum shearing strain on the $z$ plane is

$$
\gamma=\gamma_{x} v^{\prime}=\epsilon_{x}-\epsilon_{y}=\frac{2 z}{h} \gamma_{1},
$$

where $\gamma_{1}$ is the shearing strain at the extreme fiber $\gamma_{1}=\epsilon_{1}-\epsilon_{2}$. Because of the antisymmetry of the deformation $\epsilon_{1}=-\epsilon_{2}$, so that

$$
\gamma_{1}=2 \epsilon_{1} \text {. }
$$

The shearing stress corresponding to the strain $\gamma$ is given by

$$
\tau=G \gamma
$$

nside the elastic range. The twisting moment per unit length is given by

$$
m_{x y^{\prime}}=\int_{-h / 2}^{h / 2} \tau z d z \text {. }
$$

Substituting (1), (7), (5), and (6) in this equation gives

$$
\frac{P}{2}=\frac{1}{3} \epsilon_{1} G h^{2} \text {. }
$$

From which

$$
G=\frac{3}{2 h^{2}} \frac{P}{\epsilon_{1}} .
$$

Hence the shear modulus can be computed from the slope $P / \epsilon_{1}$ of a plot of load against extreme fiber strain by substituting $P / \epsilon_{1}$ in (10)

The shear modulus may be determined also from the deflection $w_{a}$ of the center of the plate relative to a line connecting points a distance $a$ from the center along one of the diagonals, figure 2 . If plane sections are assumed to remain plane, the curvature $1 / R$ along the diagonal $x$ is given by the familiar formula

$$
\frac{1}{R}=\frac{d^{2} w}{d x^{2}}=\frac{\epsilon_{1}}{h / 2}
$$

where $w=$ normal deflection. Substituting (11) in (9) gives

$$
w_{a}=\frac{\epsilon_{1} a^{2}}{h}=\frac{3}{2} \frac{a^{2}}{h^{3}} \frac{P}{G},
$$

so that

$$
G=\frac{3}{2} \frac{a^{2}}{h^{3}} \frac{P}{w_{a}},
$$

which gives the desired relation between shear modulus and center deflection under a given load.

\subsection{Extension into plastic range}

Equations (1) to (6) apply beyond the elastic range as long as plane sections remain plane. Equation (8) for the relation between twisting moment and shearing stress applies beyond the elastic range also. We may replace $z$ in the integral of that equation by $\gamma$, using eq (5) so that eq (8) becomes

$$
m_{x^{\prime} y^{\prime}}=\frac{P}{2}=\frac{h^{2}}{2 \gamma_{1}^{2}} \int_{0}^{\gamma_{1}} \tau \gamma d \gamma .
$$

The change in load $d P$ corresponding to a small change in extreme fiber shearing strain $d \gamma_{1}$ is obtained by differentiating this equation in respect to $\gamma_{1}$ with the result

$$
\frac{d P}{d \gamma_{1}}=-\frac{2 h^{2}}{\gamma_{1}^{3}} \int_{0}^{\gamma_{1}} \tau \gamma d \gamma+\frac{h^{2}}{\gamma_{1}^{2}} \tau_{1} \gamma_{1}=\frac{1}{\gamma_{1}}\left(-2 P+h^{2} \tau_{1}\right),
$$

where $\tau_{1}$ is the extreme fiber shearing stress. Solving this equation for $\tau_{1}$ gives

$$
\tau_{1}=\frac{1}{h^{2}}\left[\gamma_{1} \frac{d P}{d \gamma_{1}}+2 P\right]
$$

The extreme fiber shearing strain can be computed simply from measured values of extreme fiber bending strain $\epsilon_{1}$ or from measured values of deflection $w_{a}$ by making use of eq (6) and (12)

$$
\gamma_{1}=2 \epsilon_{1}=\frac{2 h w_{a}}{a^{2}}
$$


The stress-strain curve in shear can be derived, therefore, from measurements of $P\left(\epsilon_{1}\right)$ or $P\left(w_{a}\right)$ by using (17) to compute the shearing strain $\gamma_{1}$, plotting $P\left(\gamma_{1}\right)$, and computing $\tau_{1}$ from the ordinates and slope of $P\left(\gamma_{1}\right)$ in accordance with eq $(16)$.

It is interesting to note that eq (16) is of the same form as the equation

$$
\sigma_{1}=\frac{2}{b h^{2}}\left[\epsilon_{1} \frac{d M}{d \epsilon_{1}}+2 M\right]
$$

which is derived by Nadai [6] to compute the stressstrain curve $\sigma_{1}\left(\epsilon_{1}\right)$ from the bending moment-strain curve $M\left(\epsilon_{1}\right)$ for a rectangular beam of width, $b$, and height, $h$, in pure bending; $\epsilon_{1}$ is the extreme fiber bending strain produced by the bending moment, $M$. The identity in form of eq (16) and (18) might be expected because the square plate is bent plastically into an anticlastic surface by equal and opposite bending moments per unit length about axes at right angles to each other.

\section{Design of Specimen}

In designing the specimen, consideration must be given to practical deviations from the theoretical behavior because the theory neglects edge effects, membrane stresses, and deformation by transverse shearing forces. These effects must be considered in some detail in order to dimension the specimen so that the deviations from ideal behavior can be either neglected or taken into account.

The edge effect will be considered first. In the theory, the concentrated forces at the corners are replaced by twisting moments uniformly distributed along each edge, which are applied by horizontal shearing forces rather than the vertical forces shown in figure 2. It follows that the theoretical solution will hold only for regions of the plate at a sufficient distance from the edges, to make Saint-Venant's principle applicable. Dimensional reasoning indicates that the "sufficient distance" should be proportional to the thickness, $h$. The factor of proportionality may be estimated roughly by examining cases for which solutions have been obtained from the theory of elasticity.

The plane stress solution for a bar of constant width $h$ compressed by a concentrated load at the center of the free ends is given in [7]. This shows that the maximum compressive stress, which becomes infinite directly under the load, differs less than 3 percent from the average stress at a distance $h$ from the end. Seewald's plane stress analysis of the local stresses near a concentrated transverse load acting at the center of a slender beam of rectangular section with simple supports at the ends [8] shows that these stresses become negligible compared to the extreme fiber bending stresses derived from simple beam theory at a distance from the load greater than the depth of beam.

This suggests that the errors resulting from the application of Saint-Venant's principle will be small in regions of the plate at a distance from the edge

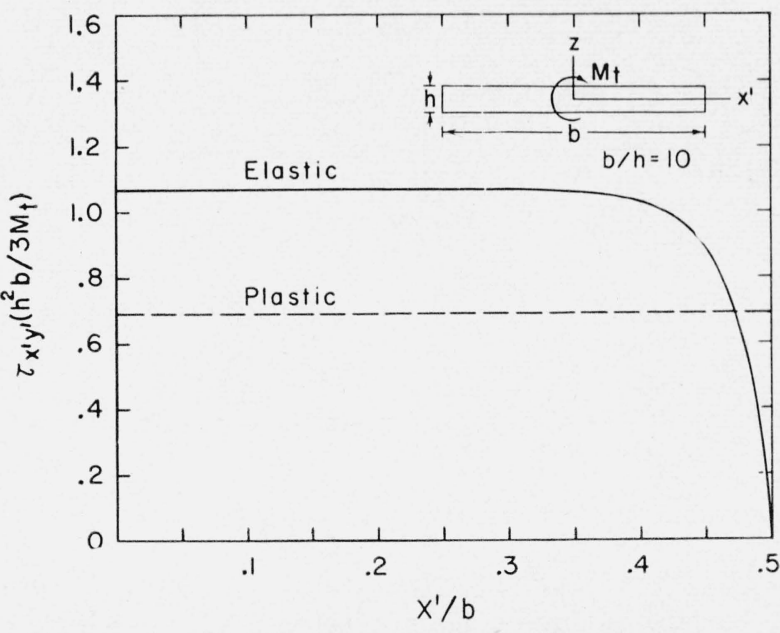

FIgURE 4. Distribution of extreme fiber shear stress $\left(\tau_{x^{\prime} y^{\prime}}\right)_{\max }$ along surface of strip with $b / h=10$.

which is greater than $h$. This condition is easily satisfied for a plate that is thin in relation to its lateral dimensions. Unfortunately, it will be shown below that a very thin plate is not acceptable because it will develop appreciable membrane stresses under the loads required to produce yielding in shear. The membrane stresses are negligible only if the plate has a width not exceeding a few times the thickness. In such a plate, it is probable that the deviations from the assumed stress distribution at the edges will have an appreciable effect upon the magnitude of the stresses in the central portion of the specimen.

A correction for the edge effect in the twisted square plate was suggested to the authors by their associate, Samuel Levy. He pointed out that the twisting of an elastic square plate can be considered as a special case of torsion of a rectangular plate considered by Kelvin and Tait [9]. The elastic stress distribution at crosssections of a long rectangular plate far from the ends is given in [10]. The extreme fiber shearing stress is plotted in figure 4 for the case $b / h=10$ which was chosen, after a preliminary analysis, as a practical compromise for the twisted plate. Kelvin and Tait [11] showed that the shearing stresses $\tau_{x^{\prime} y^{\prime}}$ parallel to the plane of the strip resist only one-half of the external twisting moment $M_{t}=P b$ transmitted by each transverse section of the strip. The other half is resisted by the transverse shearing stresses $\tau_{y^{\prime} z}$ acting on the section.

A redistribution of stresses will take place as the strip is twisted beyond the elastic range. The nature of this redistribution becomes clear by considering the extreme case of stress in a twisted strip of perfectly plastic material when all the material has yielded. This case was solved by Nadai [12] with the sand heap analogy. The resultant stress distributions are shown dotted in figures 4 and 5 . Integration of the stresses for the perfectly plastic state shows that, in the plastic as well as the elastic range, $M_{t} / 2$ is resisted by the shearing stresses $\tau_{x^{\prime} y^{\prime}}$, parallel to the surface of the strip and $M_{t} / 2$ by the 


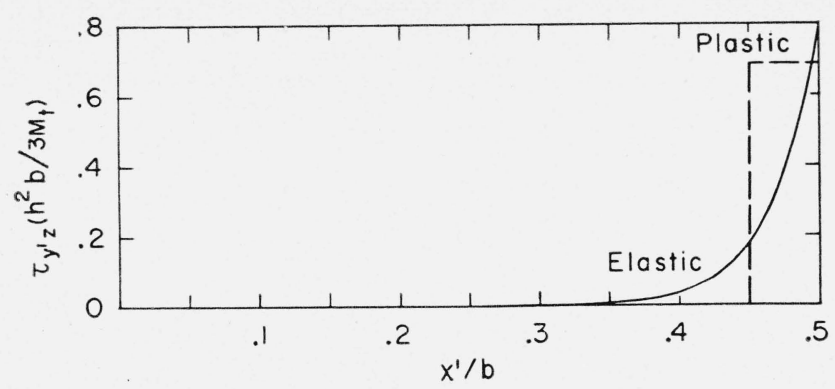

Figure 5. Distribution of transverse shear stress $\left(\tau_{y^{\prime} z}\right)_{\max }$ along $x^{\prime}$-axis of strip with section $b / h=10$.

shearing stresses $\tau_{y^{\prime} z}$ normal to the surface of the strip.

The shear stress distribution of figures 4 and 5 and the condition that one-half of the twisting moment $M_{t}$ is resisted by $\tau_{x^{\prime} y^{\prime}}$ and the other by $\tau_{y^{\prime} z}$ show that the stresses across transverse sections of the strip can be approximated by application of equal and opposite concentrated forces near the ends of the section and a uniformly distributed twisting moment along the entire section.

This distribution of equivalent external forces coincides with that shown in figure 2 as acting on the edge $y^{\prime}=b / 2$ of the square twisted plate provided that the corner forces $P$ are moved in from the corners by a small distance to make $P / 2$ act at the same point as the resultant of the transverse shearing stresses $\tau_{y^{\prime} z}$ which are shown in figure 5 for points along the $x^{\prime}$-axis. It may be concluded that the distribution of forces acting on two edges of the square plate in figure 2 nearly balances the shearing stresses acting across any transverse section of a long strip of width, $b$, and thickness, $h$, which is twisted by a torque $M=P b$ applied to the ends. The square plate can be regarded, therefore, as a portion of such a long twisted strip with a distribution of shearing stress across the section $y^{\prime}=0$ which approximates that in the transverse sections of the strip. Consequently, the extreme fiber shearingstress distributions indicated in figure 4 would also apply to the square plate. The shearing stress in the central portion would be greater than that computed from eq (16) because of the above-mentioned edge effect. In the elastic case, this increase would be about 7 percent for $b / h=10$, according to figure 4 .

In general, we find from [10] that the extreme fiber shearing stress in the central portion of the longer sides of a rectangular section can be expressed by

$$
\tau_{1 c}=\frac{\tau_{1}}{3 k_{2}}
$$

where $k_{2}$ is a factor depending on $b / h$. For values of $b / h>5$, the relationship reduces to

$$
\frac{\tau_{1 c}}{\tau_{1}}=\frac{1}{1-0.63 h / b} .
$$

In the limiting case of a perfectly plastic material which has yielded throughout the section, we have from the sand-heap analogy [12]

$$
\frac{\tau_{1 c}}{\tau_{1}}=\frac{1}{1-0.333 h / b} \cdot
$$

For $b / h=10$, we obtain from (20) and (21)

$$
\left.\begin{array}{ll}
\tau_{1 c}=1.067 \tau_{1} & \text { elastic case } \\
\tau_{1 c}=1.034 \tau_{1} & \text { plastic case }
\end{array}\right\} \text {. }
$$

The actual correction for edge effect beyond the elastic range should lie somewhere between the limits given by eq (20) and (21). It should be closer to (20) as long as the plastic yielding is localized in the outer fibers of the plate.

The effect of membrane stresses will be considered next. The stiffening effect of membrane stresses is noticeable experimentally during twisting of a thin square plate as the transverse displacements $w(x, y)$ become comparable to the thickness of the plate. Figure 6, taken from Figure 11 of [13] shows the effect for a $7.2 \times 7.2 \times 0.126$-in. plate of $24 \mathrm{~S}-\mathrm{T}$ aluminum alloy. The two straight lines in the figure are the strains $\epsilon_{1}$ along the diagonals computed from eq (9) for the linear elastic case. It is seen that the measured load $P$ agrees closely with the calculated values at very small strains, but that it deviates increasingly with increasing strains. The deviation is close to 30 percent for a strain $\epsilon_{1}=0.0008$.

The effect is analyzed in the appendix for the elastic range on the assumption that the transverse deflection of the plate continues to be given by eq (3). Equation (67) in the appendix shows that

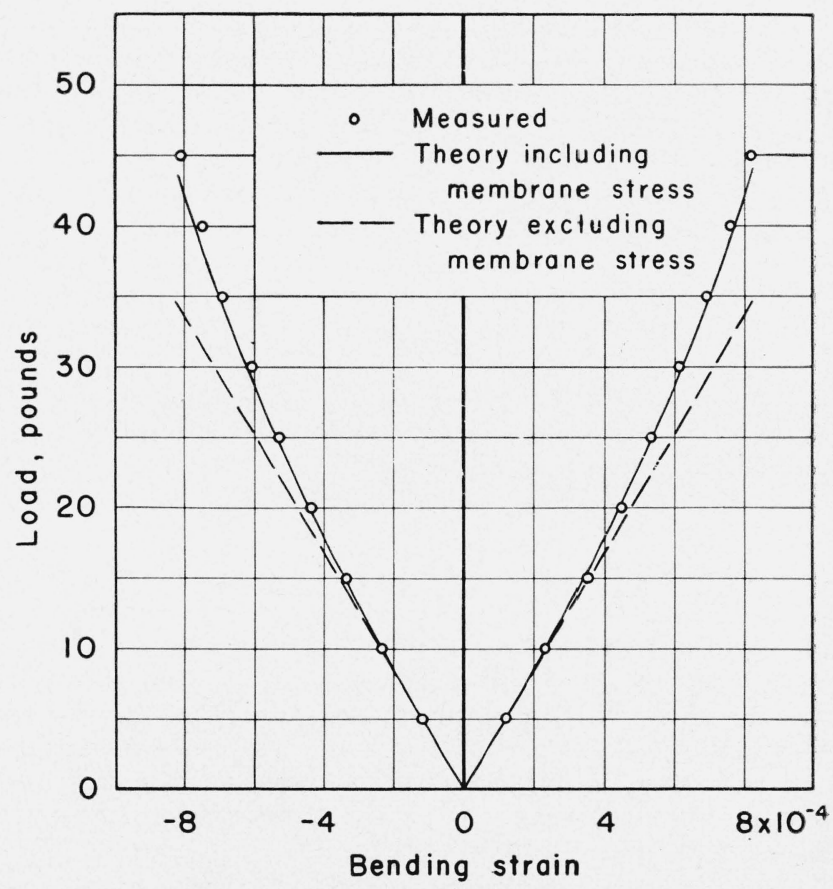

FiguRE 6. Extreme fiber bending strains at center and along diagonals of $7.2 \times 7.2 \times 0.126 \mathrm{in}$. plate of aluminum alloy 24S-T twisted by transverse forces $P$ applied at the corners. 
the relative error in extreme fiber bending strain $\epsilon_{1}$ caused by the stiffening effect, is equal to

$$
\frac{\Delta \epsilon_{1}}{\epsilon_{1}}=0.03666\left(\frac{b}{h}\right)^{4} \epsilon_{1}^{2} .
$$

The error goes up with the square of the extreme fiber strain $\epsilon_{1}$ and with the fourth power of the width/ thickness ratio, $b / h$. It may be made small by making $b / h$ small.

Lastly, consideration will be given to the transverse shearing stress. The effect of transverse shearing stress will be negligible as long as this stress is very small compared to the extreme fiber shearing stress parallel to the plane of the plate. The average transverse shearing stress $\tau_{\mathrm{r}}$ on a section of a cylinder at a radius, $r$, from one of the corners of the plate (fig. 2) is

$$
\tau_{r}=\frac{P}{\pi r h / 2} .
$$

The extreme fiber shearing stress in the elastic range is from (7), (6), and (9)

$$
\tau_{1}=G \gamma_{1}=2 G \epsilon_{1}=3 P / h^{2} .
$$

The ratio of the average transverse shearing stress to the extreme fiber shearing stress in the elastic range becomes

$$
\frac{\tau_{r}}{\tau_{1}}=\frac{2}{3 \pi} \frac{h}{r}=0.2 \frac{h}{r} .
$$

This requires $h / r$ to be small, that is, the effect becomes negligible as the plate is made very thin.

Equations (20), (23), and (26) were used to estimate the deviations from ideal behavior caused by edge effect, membrane strain, and transverse shear for square plates with width/thickness ratios $b / h=5$,

\begin{tabular}{|c|c|c|c|}
\hline & \multicolumn{3}{|c|}{$b / h$} \\
\hline & 5 & 10 & 20 \\
\hline $\begin{array}{l}\text { Edge effect }\left(\tau_{1 c} / \tau_{1}\right)-1 \\
\text { Membrane stiffening effect } \Delta \epsilon_{1} / \epsilon_{1} \\
\text { Transverse shear effect } \tau_{r} / \tau_{1}\end{array}$ & $\begin{array}{r}0.13 \\
.00 \\
.20\end{array}$ & $\begin{array}{r}0.07 \\
.02 \\
.10\end{array}$ & $\begin{array}{r}0.03 \\
.38 \\
.05\end{array}$ \\
\hline
\end{tabular}
10,20 . The results, rounded off to the nearest percent, are given in table 1.

TABLE 1 .

In table $1\left(\tau_{1 c} / \tau_{1}\right)-1$ is taken as the correction according to eq (20) for the elastic case. The extreme fiber shearing strain $\gamma_{1}=2 \epsilon_{1}$ is assumed as 0.016 to place it well beyond the elastic range. The membrane stiffening effect was computed from eq (23) for the elastic range, since the stiffening will be relatively insensitive to the vielding that takes place near the extreme fibers of the twisted plate. The average transverse shearing stress was computed for a radius $r=b / 5$ from the corner leaving the inner portion of the plate $0.2<r / b<0.7$ available for strain and displacement measurements.

Table 1 indicates that a plate with $b / h=10$ provides a fair compromise between the three different disturbing effects that were considered.

\section{Tests}

\subsection{Test procedure}

Specimens were made from aluminum alloy $75 \mathrm{~S}-\mathrm{T} 6$ sheet nominally 0.125 in. thick for which the tensile and compressive stress-strain curves had been obtained both parallel and perpendicular to the direction of rolling [14]. This material was selected because of its approximately isotropic properties. The specimens were taken from near the center of the sheet, where there was little variation in thickness. The specimen selected for trying out the procedure was $0.1324 \mathrm{in}$. thick and $1.25 \mathrm{in}$. square. This gave a width/thickness ratio $b / h=9.44$, which approximates the value $b / h=10$ suggested in the previous section. The specimen was large enough to provide space for mounting a deflectometer and for attaching strain gages at several locations. It had additional material at each corner, as shown in figure 7 , to reduce the concentration of transverse shearing stress at the corners. A conical seat was machined at each corner of the square, a pair on one side at the ends of a diagonal and a pair on the other side at the ends of the other diagonal.

The specimen $\mathrm{S}$, figure 8, was loaded through $1 / 8$-in. steel balls in the conical seats at the corners with a beam-and-poise screw-type testing machine of 50,000-lb capacity used in the 5,000-lb range. In this manner of loading, the load acted along vertical lines through the centers of the balls, which were almost a thickness of the sheet from the neutral surface. This resulted in the moment becoming larger instead of less as the specimen twisted. This effect is opposite in sign to the stiffening effect of membrane stresses previously mentioned. Two corners of the specimen rested upon balls. One of these was seated in a block of steel resting on the upper head of the testing machine, and the other was seated in the block $A$, figure 8 , which was free to move on

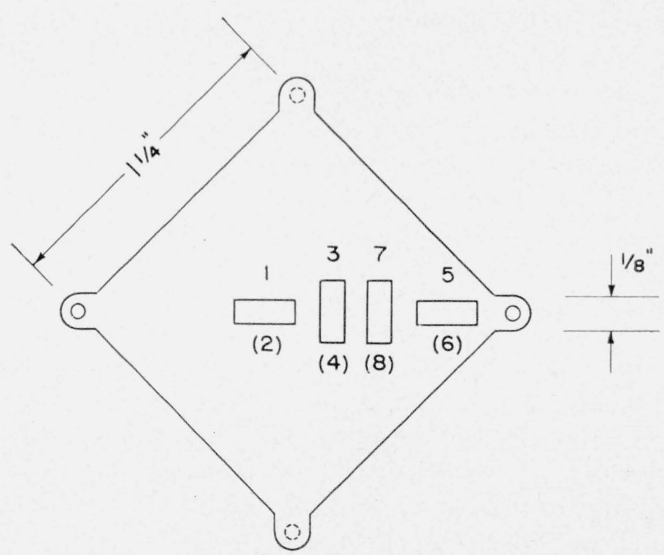

Figure 7. Location of wire strain gages on plate. 


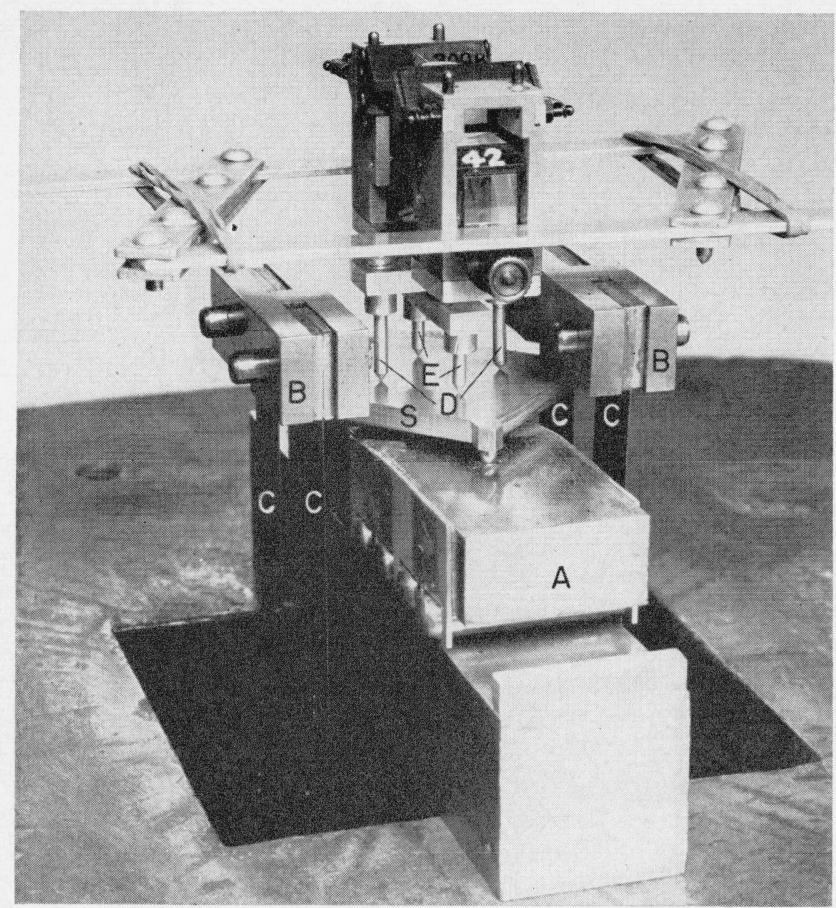

Figure 8. Plate under load with deflectometer in position for measuring deflections along principal diagonals.

rollers in the direction of the diagonal. The other two corners were loaded by means of a hanger connected to the loading head of the machine by a rod having a ball-and-socket joint at each end. The upper cross pieces, B, in which the balls were seated are shown in figure 8 . They were connected to a common piece below the head by steel tapes, C. The ball joints at each end of the rod and the pin joints at each end of the tapes had the effect of equalizing the load on the corners of the plate.

SR -4 type A-7 strain gages were mounted on the specimen in pairs on opposite sides of the specimen. The gages were connected with an SR-4 model K portable strain indicator through a multiple-pole mercury-contact switch. A gage cemented to a piece of the sheet material was used as a temperature compensating gage. Since each portion of the specimen was subject to both tensile and compressive strains at right angles to each other, the gage-factor setting was taken as the axial sensitivity of the gage computed from the gage factor provided by the manufacturer and the data on axial and transverse sensitivities for gages of this type [15]. Strain readings were estimated to 0.1 division $(0.000001)$. A correction was applied to compensate for the strain sensitive wire being farther from the neutral surface of the specimen than the surface of the sheet; each strain was multiplied by the ratio of the thickness of the specimen to the sum of the thicknesses of the specimen and the gage.

A deflectometer using two Tuckerman optical strain gages with 0.4 -in. lozenges, figure 8 , was used to measure deflections relative to the center of the plate. The Tuckerman strain gages were mounted so that their knife edges were connected to a pair of legs, D, placed along one diagonal of the plate, while their lozenges were connected to a pair of shorter legs, E, placed along the other diagonal. One of the gages was cemented to the cross piece holding the two longer legs. The knife edge of the other gage was mounted on a 1/4-in. tube which was inserted in the cross piece midway between the two legs. The lozenges were actuated by a 1/4-in. rod, which was screwed into the cross piece holding the two shorter legs and which was reduced at the lower end to pass through the tube with ample clearance. The lozenges were set near their lower limits of travel so that the vertical component of the clamping force would assist in maintaining contact of the two shorter legs with the specimen. The deflectometer was mounted on the specimen symmetrically with respect to the center with the point of one of each pair of legs in a small punch mark on each diagonal and the other in a scratch along the diagonal. The span was about 0.7 in. The deflectometer measured the sum of the deflections at the center with respect to each pair of points or twice such a deflection, since the loading was equal and opposite at each pair of corners. With this deflectometer, deflections could be read to the nearest 0.000002 in.

\subsection{Test Results \\ a. Elastic Range}

Wire strain gages were cemented on the specimen at locations 1 and 3 , and 2 and 4 , respectively opposite them on the other side; figure 7 . The specimen and deflectometer were set up as shown in figure 8 , and the specimen was loaded in the elastic range. The value of the shear modulus is from (6), (12)

$$
G=\frac{\tau_{1 c}}{\gamma_{1}}=\frac{\tau_{1 c}}{2 \epsilon_{1}}=\frac{\tau_{1 c} a^{2}}{2 w_{a} h}
$$

where $\tau_{1 c}$ is the shearing stress after correction for edge effect. Within the elastic range, from (20) and $(25)$

$$
\tau_{1 c}=\frac{3 P}{h^{2}(1-0.63 h / b)}
$$

or with $b / h=9.44$,

$$
\tau_{1 c}=3.21 P / h^{2} .
$$

Substitution of $\tau_{1 c}$ in $(27)$ gives the shear modulus in terms of either measured extreme fiber strain $\epsilon_{1}$, or measured center deflection $w_{a}$. Table 2 shows the values thus obtained from the initial linear portion of plots of deflectometer readings and wire strain-gage readings as a function of load $P$.

The last value of $G$ in table 2 was computed from

$$
G=\frac{E}{2(1+\nu)},
$$

by substituting for the Young's modulus an average 
TABLE 2. Shear-modulus values measured at central portion of square plate

\begin{tabular}{|c|c|}
\hline Obtained from- & $G$ \\
\hline $\begin{array}{l}\text { Deflectometer } \\
\text { Strain gage 1 } \\
\text { Strain gage 2 } \\
\text { Strain gage } 3 \\
\text { Strain gage } 4 \\
\text { Equation }(30)\end{array}$ & $\begin{array}{l}\text { lb/in. } .^{2} \\
3.82 \times 10^{6} \\
3.87 \\
3.82 \\
3.84 \\
3.88 \\
3.90\end{array}$ \\
\hline
\end{tabular}

value $E=10.37 \times 10^{6} \mathrm{lb} / \mathrm{in}^{2}{ }^{2}$ obtained from tensile and compressive tests of the aluminum alloy $75 \mathrm{~S}-\mathrm{T} 6$ sheet, and for Poisson's ratio $\nu=0.33$, the nominal value for this alloy.

Table 2 shows that the experimental values of shear modulus agreed within 1.5 percent. The average for the wire gages was about 0.7 percent greater than the value obtained from the deflection data and about 1.2 percent less than the value computed from eq (30). A lower value from the deflection data would be expected because of the effect of transverse shear.

The deflectometer was removed, and gages 5 and 7 , figure 7 , and gages 6 and 8 , respectively opposite them, were cemented to the specimen. The loading was repeated in the elastic range. The results are given in table 3 .

TABLE 3. Shear modulus values measured in outer portion of square plate

\begin{tabular}{|c|c|}
\hline $\begin{array}{c}\text { Obtained } \\
\text { from gages }\end{array}$ & $G$ \\
\hline $\begin{array}{l}5 \\
6 \\
7 \\
8\end{array}$ & 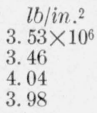 \\
\hline
\end{tabular}

The results show greater strains at locations 5 and 6 than at any other location. This indicates a deviation from the uniform state of stress, which becomes serious in the corner portions of the plate.

\section{b. Plastic range}

The specimen was loaded until a shear strain of about 2 percent was reached at the extreme fiber. Deflections were measured with the deflectometer and strain with the four central wire gages. The shear stress was partially corrected for edge effect by substitution of $\tau_{1}$, eq (16), in eq (20). Equation (20) was used beyond the elastic range to avoid discontinuity at the end of the elastic range. It should be an adequate approximation in the important region near the knee of the stress-strain curve because only a small proportion of the material would be in the plastic range.

The stress-strain data are plotted in figure 9 . The values of $G$ determined both from the strain data and deflection data were a little over 2 percent higher than in the first test, the average value for the four wire gages being almost 1 percent above the value computed from (30). No explanation could be found for this small deviation.

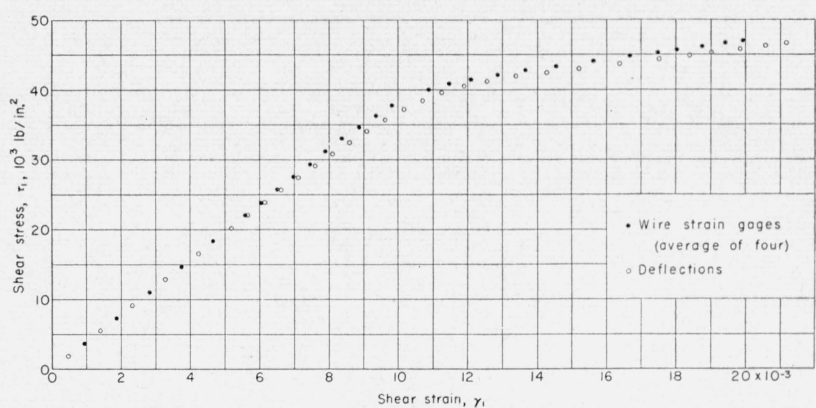

Figure 9. Stress-strain curve in shear, aluminum alloy $75 \mathrm{~S}-\mathrm{T} 6$ sheet.

c. Comparison with stress-strain curves under axial load

It is interesting to compare the stress-strain curve in shear with that under axial load. Such a comparison can be made by deriving the octahedral stressstrain curve in each case [16]. The octahedral stressstrain curves for both states of stress should coincide if the maximum shear strain $\gamma_{o}$ on the octahedral plane (the plane defined as having a normal which makes equal angles with the three axes of principal stress) is a single-valued function of the maximum shearing stress $\tau_{o}$ on the octahedral plane, regardless of the direction and proportion of the principal stresses.

The octahedral stress-strain curve $\tau_{o}\left(\gamma_{o}\right)$ was computed from an average axial stress-strain curve $\sigma(\epsilon)$ by substitution in the following formulas (16):

$$
\tau_{o}=\frac{\sqrt{2}}{3} \sigma ; \quad \gamma_{o}=\frac{\tau_{o}}{G}+\sqrt{2}\left(\epsilon-\frac{\sigma}{E}\right) .
$$

The axial stress $\sigma$ was obtained from tensile and compressive stress-strain curves of the material parallel to and perpendicular to the direction of rolling [14] as the average of the tensile and compressive stresses corresponding to a given axial strain $\epsilon$. The value of $G$ used, $3.93 \times 10^{6} \mathrm{lb} / \mathrm{in}^{2}$, was the average determined from the strain data in this test. The value of $E$ used, $10.37 \times 10^{6} \mathrm{lb} / \mathrm{in}^{2}{ }^{2}$, was the average obtained from tensile and compressive tests on the sheet. The stress-strain curve in shear determined from the strain data, figure 9, was converted similarly into an octahedral stress-strain curve by substitution in

$$
\tau_{o}=\sqrt{\frac{2}{3}} \tau_{1} ; \quad \gamma_{o}=\frac{\tau_{o}}{G}+\sqrt{\frac{2}{3}}\left(\gamma_{1}-\frac{\tau_{1}}{G}\right) .
$$

The resulting curves of octahedral stress versus total strain $\gamma_{o}$ and versus permanent strain $\gamma_{0}-\tau_{o} / G$ are plotted in figure 10 . It will be noted that the two stress-strain curves are in good agreement except for deviations up to 5 percent near the knee and at shearing strains exceeding 0.009 .

In this connection, mention should be made of the much larger discrepancies found in exploratory twisting tests of a $0.125 \times 0.625 \times 0.625$-in. plate of SAE 1025 steel. These tests were made to extend the above comparison to a material with a pro- 


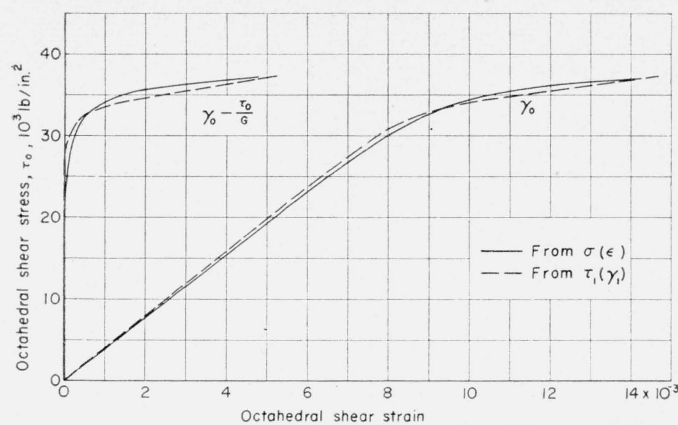

Figure 10. Octahedral stress-strain curve, aluminum alloy $75 \mathrm{~S}-\mathrm{T} 6$ sheet.

nounced yield point. The shear modulus was found to be in good agreement with the value computed from Young's modulus and Poisson's ratio. However, the octahedral stress corresponding to the yield point obtained from the twisting test was 38 percent greater than that obtained from the average of the yield points of tensile and compressive specimens of the material. Twisting tests of long strips of width/thickness ratios equal to 5 and 10 gave octahedral stresses corresponding to the yield point respectively 17 and 26 percent greater than for the average of the tensile and compressive tests. A twisting test of a long round specimen 0.59 in. in diameter, on the other hand, showed a corresponding octahedral stress 15 percent lower.

\section{Conclusion}

The stress-strain curve in shear up to strains of the order of 0.01 can be obtained for isotropic flat sheet from measurements of deflection or of extreme fiber bending strain in the center portion of a twisted square plate, provided that a correction is made for the edge effect. The shear moduli obtained from the twisting test of a $0.13 \times 1.25 \times 1.25$-in. square plate of $75 \mathrm{~S}-\mathrm{T}$ aluminum alloy agreed within 2 percent with the value calculated from the average of the tensile and compressive moduli and an accepted value of Poisson's ratio. The octahedral stress derived from the stress-strain curve in shear for the twisted plate agreed within about 3 percent with the octahedral stress derived from tensile and compressive tests of the same material except near the knee, where the systematic deviations were as high as 5 percent. Much greater deviations were found in exploratory tests of mild steel.

Unfortunately, the method is difficult to periorm on sheet material because the specimen must be small in size. Alternative methods should be investigated. An examination of other methods indicates that the annulus of constant thickness method can be used for determining the stress-strain curve in shear, even in the presence of a large radial variation in shear stress. Preparations are under way for carrying out such tests at the National Bureau of Standards.

\section{Appendix}

\subsection{Membrane stresses in elastic range}

The membrane stresses in a twisted square plate can be estimated from the equations for the state of strain in a flat plate with large deflections. These are given by Love [17] as

$$
\left.\begin{array}{c}
\epsilon_{x}=\frac{\partial u}{\partial x}+\frac{1}{2}\left(\frac{\partial w}{\partial x}\right)^{2} \\
\epsilon_{y}=\frac{\partial v}{\partial y}+\frac{1}{2}\left(\frac{\partial w}{\partial y}\right)^{2} \\
\gamma_{x y}=\frac{\partial v}{\partial x}+\frac{\partial u}{\partial y}+\frac{\partial w}{\partial x} \frac{\partial w}{\partial y}
\end{array}\right\} .
$$

It is assumed that the plate of figure 2 is bent into an anticlastic surface so that the transverse deflection is given by

$$
w=\frac{x^{2}-y^{2}}{2 R}
$$

just as in the case of small deflections, eq (3).

The curvature is from (11)

$$
\frac{1}{R}=\frac{2 \epsilon_{1}}{h}
$$

The magnitude of the membrane strain depends on the displacements $u, v$ parallel to the $x, y$ axes as well as on $w$.

The displacement $u$ in the $x$-direction can be approximated by a polynomial of the form

$$
u=\sum_{i, j} c_{i j} x^{i} y^{j}
$$

where $i, j$ are positive integers. The coefficients $c_{i j}$ of many of the terms in the series must be equal to zero in order to satisfy the following conditions of symmetry and antisymmetry in the plate, figure 2 :

$$
\left.\begin{array}{l}
u(x, y)=u(x,-y) \\
u(x, y)=-u(-x, y)
\end{array}\right\} .
$$

These conditions are satisfied if $i, j$ are confined to

$$
\left.\begin{array}{l}
i=1,3,5 \ldots \\
j=0,2,4 \ldots
\end{array}\right\} \text {. }
$$

The displacement $v$ at the point $(y, x)$ must be equal to the displacement $u$ at the point $(x, y)$, so that

$$
v=\sum_{i, j} c_{i j} y^{i} x^{j}
$$


Up to and including terms of the fifth power, the series (36) and (39) become

$$
\left.\begin{array}{l}
u=c_{10} x+c_{12} x y^{2}+c_{30} x^{3}+c_{14} x y^{4}+c_{32} x^{3} y^{2}+c_{50} x^{5} \\
v=c_{10} y+c_{12} x^{2} y+c_{30} y^{3}+c_{14} x^{4} y+c_{32} x^{2} y^{3}+c_{50} y^{5}
\end{array}\right\} .
$$

To satisíy the conditions that the membrane stresses $\sigma_{x^{\prime}}, \tau_{x^{\prime} y^{\prime}}$ be zero along the edge

$$
y=\frac{b}{\sqrt{2}}-x
$$

of the plate, and that the strain energy stored in the form of membrane stress must be a minimum, the six unknowns $c_{10}, c_{12}, c_{30}, c_{14}, c_{32}$, and $c_{50}$ were determined as follows.

Equilibrium of forces on an element of the plate bounded by the edge and by planes $y=$ const., $x=$ const. shows that the membrane stresses along the edge are related to the membrane stresses $\sigma_{x}, \sigma_{y}, \tau_{x y}$ by

$$
\left.\begin{array}{r}
\sigma_{x^{\prime}}=\frac{\sigma_{x}+\sigma_{y}}{2}+\tau_{x y} \\
\tau_{x^{\prime} y^{\prime}}=\frac{\sigma_{x}-\sigma_{y}}{2}
\end{array}\right\}
$$

The stresses $\sigma_{x}, \sigma_{y}, \tau_{x y}$ must be adjusted to make $\sigma_{x^{\prime}}$ and $\tau_{x^{\prime} y^{\prime}}$ in $(42)$ equal to zero. The membrane stresses are related to the membrane strains $\epsilon_{x}, \epsilon_{y}$, $\gamma_{x y}$ by Hooke's Law

$$
\left.\begin{array}{rl}
\sigma_{x} & =\frac{E}{1-\nu^{2}}\left(\boldsymbol{\epsilon}_{x}+\nu \boldsymbol{\epsilon}_{y}\right), \quad \sigma_{y}=\frac{E}{1-\nu^{2}}\left(\boldsymbol{\epsilon}_{y}+\nu \epsilon_{x}\right) \\
\tau_{x y} & =\frac{E}{2(1+\nu)} \gamma_{x y}
\end{array}\right\},
$$

where E, $\nu$ are Young's modulus and Poisson's ratio, respectively.

The membrane strains may be computed from the displacements, using eq (33). Carrying out the computations and using the following notation for convenience

$$
\left.\begin{array}{c}
\xi=\frac{x}{b} ; \quad \eta=\frac{y}{b} ; \quad \rho=\begin{array}{l}
b \\
R
\end{array} \\
c_{0}=c_{10} ; \quad c_{2}=c_{12} b^{2} ; \quad c_{3}=3 c_{30} b^{2}+\frac{b^{2}}{2 R^{2}} \\
c_{4}=c_{14} b^{4} ; \quad c_{5}=c_{50} b^{4} ; \quad c_{3}=c_{32} b^{4}
\end{array}\right\}
$$

lead to

$$
\left.\begin{array}{rl}
\epsilon_{x} & =c_{0}+c_{2} \eta^{2}+c_{3} \xi^{2}+c_{4} \eta^{4}+3 c_{6} \xi^{2} \eta^{2}+5 c_{5} \xi^{4} \\
\epsilon_{y} & =c_{0}+c_{2} \xi^{2}+c_{3} \eta^{2}+c_{4} \xi^{4}+3 c_{6} \xi^{2} \eta^{2}+5 c_{5} \eta^{4} \\
\gamma_{x y} & =\left[4 c_{2}-\rho^{2}+\left(4 c_{4}+2 c_{6}\right)\left(\xi^{2}+\eta^{2}\right)\right] \xi \eta
\end{array}\right\} .
$$

Substituting (45) in (43) and (43), in (42) and using the notation

$$
\alpha=\frac{1-\nu}{1+\nu}
$$

gives, for the normal and shearing stresses acting on the edges,

$$
\left.\begin{array}{rl}
\sigma_{x^{\prime}}= & \begin{array}{l}
E \\
2(1-\nu)
\end{array}\left\{2 c_{0}+\left(c_{2}+c_{3}\right)\left(\xi^{2}+\eta^{2}\right)+\right. \\
& \left(c_{4}+5 c_{5}\right)\left(\xi^{4}+\eta^{4}\right)+6 c_{6} \xi^{2} \eta^{2}+\alpha \xi \eta\left[4 c_{2}-\rho^{2}+\right. \\
& \left.\left.\left(4 c_{4}+2 c_{6}\right)\left(\xi^{2}+\eta^{2}\right)\right]\right\} \\
\tau_{x^{\prime} y^{\prime}}= & \frac{E}{2(1+\nu)}\left(\xi^{2}-\eta^{2}\right)\left[\left(c_{3}-c_{2}\right)+\left(5 c_{5}-c_{4}\right)\left(\xi^{2}+\eta^{2}\right)\right]
\end{array}\right\} .
$$

The shearing stresses $\tau_{x^{\prime} y^{\prime}}$ will be zero for all points $\xi, \eta$ along the edge of the plate only if the expression in the square brackets is equal to zero. This requires that

$$
c_{3}=c_{2} ; \quad 5 c_{5}=c_{4} \text {. }
$$

The normal stress $\sigma_{x^{\prime}}$ along the edge can be expressed as a polynomial of the fourth order in $\xi$ by noting from (41) that, for points along the edge of the plate,

$$
\eta=\frac{1}{\sqrt{2}}-\xi
$$

Collecting terms by powers of $\xi$ and substituting (48) leads to

$$
\begin{aligned}
\frac{2(1-\nu)}{E} \sigma_{x^{\prime}}= & 2 c_{0}+c_{3}+\frac{5}{2} c_{5}+\frac{\xi}{\sqrt{2}}\left[-4 c_{3}-20 c_{5}+\right. \\
& \left.\alpha\left(4 c_{3}+10 c_{5}+c_{6}-\rho^{2}\right)\right]+\xi^{2}\left[4 c_{3}+30 c_{5}+\right. \\
& \left.3 c_{6}+\alpha\left(-4 c_{3}-30 c_{5}-3 c_{6}+\rho^{2}\right)\right]+ \\
& \sqrt{2} \xi^{3}\left[-20 c_{5}-6 c_{6}+\alpha\left(4 c_{6}+40 c_{5}\right)\right]+ \\
& \xi^{4}\left[20 c_{5}+6 c_{6}+\alpha\left(-4 c_{6}-40 c_{5}\right)\right]=0
\end{aligned}
$$

The stress $\sigma_{x}$, will be zero for all points $\xi$ along the edge only if each one of the five brackets on the right is equal to zero. Examination of these brackets shows that only three of them are independent of each other. One may solve the resulting three equations for any three of the coefficients $c_{0}, c_{3}$, $c_{5}, c_{6}$ in terms of the remaining coefficient.

Solving in terms of $c_{0}$ and assuming for Poisson's ratio the typical value $\nu=1 / 3$ and hence from (46) $\alpha=1 / 2$, one obtains from (50)

$$
c_{3}=-3 c_{0}+\rho^{2} / 8 ; \quad c_{5}=\frac{2}{5} c_{0}-\frac{1}{20} \rho^{2} ; \quad c_{6}=0 .
$$

Substitution of (51) and (48) in (45) shows that the 
strains reduce to

$$
\left.\begin{array}{c}
\boldsymbol{\epsilon}_{x}=\boldsymbol{\epsilon}_{y}=c_{0}+c_{3}\left(\xi^{2}+\eta^{2}\right)+5 c_{5}\left(\xi^{4}+\eta^{4}\right) \\
\gamma_{x y}=\left[4 c_{3}-\rho^{2}+20 c_{5}\left(\xi^{2}+\eta^{2}\right)\right] \xi \eta
\end{array}\right\} .
$$

It remains to compute the constant $c_{0}$ by minimizing the membrane strain energy stored in the plate. The membrane strain energy per unit volume, $V_{0}=\frac{1}{2}\left(\sigma_{x} \epsilon_{x}+\sigma_{y} \epsilon_{y}+\tau_{x y} \gamma_{x y}\right)$ is with (43) and (52), assuming. $y=1 / 3$

$$
V_{0}=\frac{3}{16} E\left(8 \epsilon_{x}^{2}+\gamma_{x y}^{2}\right)
$$

The elastic membrane strain energy $V_{m}$ stored in the plate is obtained by integrating (53) over the volume of the plate

$$
\begin{aligned}
V_{m}=4 \int_{x=0}^{b / \sqrt{2}} \int_{y=0}^{(b / \sqrt{2})-x} V_{0} h d y d x \\
=4 h b^{2} \int_{\xi=0}^{1 / \sqrt{2}} \int_{\eta=0}^{(1 / \sqrt{2})-\xi} V_{0} d \eta d \xi
\end{aligned}
$$

Substitution of (52) in (53), of the resulting expression in (54), and integration leads to

$$
\begin{aligned}
V_{m}= & \frac{3 E h b^{2}}{4}\left[2 c_{0}^{2}+\frac{2}{3} c_{0} c_{3}+\frac{1}{15}\left(c_{3}^{2}+10 c_{0} c_{5}\right)+\right. \\
& \left.\frac{1}{1440}\left(32 c_{3}^{2}-8 c_{3} \rho^{2}+\rho^{4}\right)+\frac{1}{168}\left(36 c_{3} c_{5}-c_{5} \rho^{2}\right)+\frac{13}{84} c_{5}^{2}\right]
\end{aligned}
$$

The membrane energy $V_{m}$ is minimized by computing

$$
\frac{\partial V_{m}}{\partial c_{0}}=0
$$

and substituting (51) to obtain an equation having $c_{0}$ as the only unknown. Solving for $c_{0}$ gives

$$
c_{0}=-\frac{3}{146} \rho_{2}=-0.0205 \rho^{2} .
$$

The membrane strains and stresses throughout the plate are then obtained by substituting (57) in (51) and the resulting constants in eq (47) and (52) and the latter then in (43).

The stresses can be plotted in dimensionless form by multiplying them by $h^{2} / b^{2} \gamma_{1} \tau_{1}$, where $b / h$ is the width/thickness ratio for the plate and $\gamma_{1}, \tau_{1}$ are the extreme fiber shearing strain and stress due to bending. These are from (6), (7), and (11)

$$
\gamma_{1}=\frac{h}{R} ; \quad \tau_{1}=G \frac{h}{R}
$$

The results of such computations are plotted in figure 11 as solid lines. The plots show that the membrane stress at the center of the plate consists of a uniform compressive stress

$$
\sigma_{x^{\prime}}=\sigma_{y^{\prime}}=0.082 \frac{b^{2}}{h^{2}} \gamma_{1} \tau_{1}
$$

The membrane stresses are largest at the center of the edge of the plate. The membrane normal stresses at this point are

$$
\sigma_{y^{\prime}}=0.136 \frac{b^{2}}{h^{2}} \gamma_{1} \tau_{1} ; \quad \sigma_{x^{\prime}}=0
$$

A check on this solution seemed advisable since it did not satisfy the conditions of equilibrium at all points of the plate; it would be necessary to apply a system of body forces per unit volume with components $X, Y$ in the $x, y$ directions in order to maintain equilibrium in all plate elements. The equilibrium conditions would be satisfied more nearly if $c_{0}$ is chosen to minimize the average value of $X^{2}+Y^{2}$ for the entire plate instead of minimizing the strain energy. This leads to

$$
c_{0}=-0.0187 \rho^{2}
$$

which is about 10 percent below the previous value. The membrane stresses corresponding to this value of $c_{0}$ are shown as dotted lines in figure 11. They differ less than 10 percent from the stresses previously obtained.

A second check on the adequacy of the theory was obtained by computing from it the nonlinear relation between load $P$ and extreme fiber bending strain $\epsilon_{1}$ and comparing it with the experimental values given in figure 6 . The relation between $P$ and $\epsilon_{1}$ for the elastic range was obtained by equating the increments of work done by the corner loads $d W_{P}$ and the

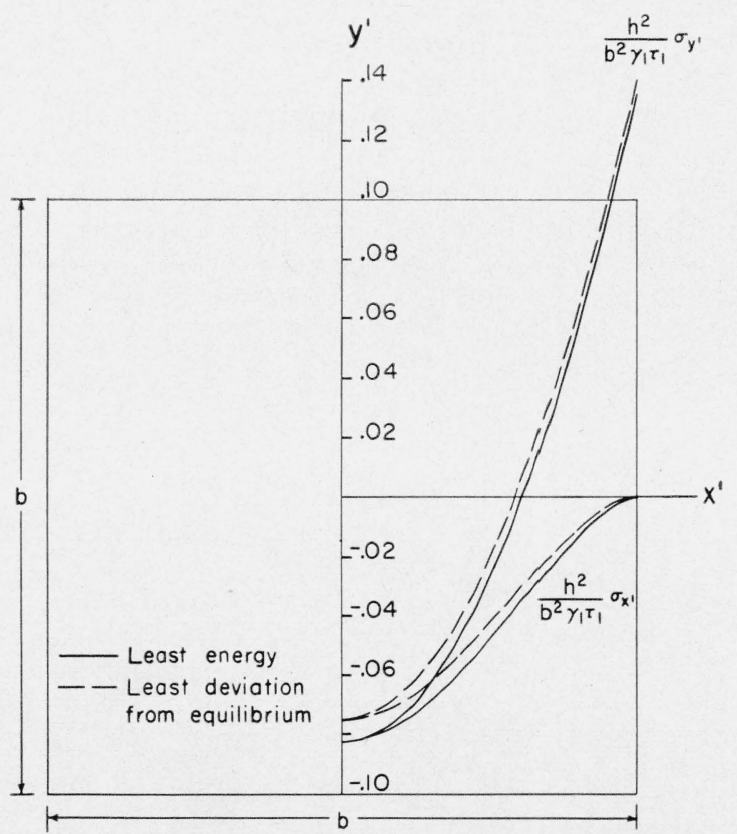

Figure 11. Elastic membrane stresses in twisted square plate. 
resulting increase in strain energy $d V$ consisting of the increment $d V_{b}$ stored in the form of bending strains and the increment $d V_{m}$ stored in the form of membrane strains

$$
d W_{P}=d V_{b}+d V_{m} .
$$

Each one of the three terms in this equation can be regarded as a function of the curvature ratio $\rho=b / R$. It can be shown easily that the work done by the corner forces $P$ corresponding to an increment of curvature ratio $d \rho$ is

$$
d W_{P}=P b d \rho .
$$

The increase in bending strain energy can be derived from the bending strain, eq (4) as

$$
d V_{b}=\frac{E h^{3}}{8} \rho d \rho
$$

The increment in membrane strain energy is obtained from (55) after substituting (57) and (51). This leads to

$$
d V_{m}=0.001146 E h b^{2} \rho^{3} d \rho .
$$

Substituting (63) to (65) in (62), solving for $P$, and replacing $\rho$ by

$$
\rho=\frac{b}{R}=2 \frac{b}{h} \epsilon_{1}
$$

gives the desired relation for $P\left(\boldsymbol{\epsilon}_{1}\right)$,

$$
P=\frac{E h^{2}}{4} \epsilon_{1}\left[1+0.03666\left(\frac{b}{h}\right)^{4} \epsilon_{1}^{2}\right] .
$$

This reduces to eq (9) for small values of $\epsilon_{1}$ with $\nu=1 / 3$. Substitution of $E=10.4 \times 10^{6} \mathrm{lb} / \mathrm{in}^{2}{ }^{2}, \quad h=$ $0.126 \mathrm{in} ., b=7.2 \mathrm{in}$. for the plate of figure 6 leads to the solid line shown in that figure. The observed points come close to this curve, thereby providing a second check on the adequacy of this approximate theory for computing membrane stresses in the elastic range.

\section{References}

[1] Ambrose H. Stang, Walter Ramberg, and Goldie Back, Torsion tests of tubes, NACA Report 601, p. 4-5, (1937).

[2] E. G. Coker and L. N. G. Filon, Photo-elasticity, p. 603 (Cambridge University Press, London, 1931).

[3] F. Bollenrath, Ausbeulerscheinungen an ebenen auf Schub beanspruchten Platten, Dissertation, Technische Hochschule, Aachen (1928).

[4] A. Nadai, Die Formaenderungen und die Spannungen von rechteckigen elastischen Platten, Forsch Gebiete Ingenieurw, Heft 170, 171, p. 16-28 (Springer, Berlin, 1915).

[5] H. W. March, E. W. Kuenzi and W. J. Kommers, Method of measuring the shearing moduli in wood, Forest Products Laboratory War Series Progress Report 1301 (June 1942)

[6] A. Nadai, Theory of flow and fracture of solids, 1 p. 359 (McGraw-Hill Book Co., Ine., New York, N. Y., 1950)

[7] S. Timoshenko and J. N. Goodier, Theory of elasticity, p. 52 (McGraw-Hill Book Co., Inc., New York, N. Y. 1951).

[8] S. Timoshenko and J. N. Goodier, Theory of elasticity, p. 102-106 (McGraw-Hill Book Co., Inc., New York, N. Y., 1951).

[9] Lord Kelvin and P. G. Tait, Natural philosophv, part 2, 2, 203 (Cambridge University Press, London, 1895).

[10] S. Timoshenko and J. N. Goodier, Theory of elasticity p. 275-278 (McGraw Hill Book Co., Inc., New York, N. Y., 1951).

[11] Lord Kelvin and P. G. Tait, Natural philosophy, part 2, 2, 267-268 (Cambridge University Press, London, 1895).

[12] A. Nadai, Theory of flow and fracture of solids, 1, p. 499 (McGraw-Hill Book Co., Inc., New York, N. Y., 1950)

13] A. E. McPherson, Device for measuring principal curvatures and principal strains on a nearly plane surface, NACA TN1137 (February 1947).

[14] James A. Miller, Stress-strain and elongation graphs for aluminum-alloy $75 \mathrm{~S}-\mathrm{T} 6$ sheet, NACA TN2085 (April 1950).

[15] William R. Campbell, Performance tests of wire strain gages, IV-Axial and transverse sensitivities, NACA TN 1042 (June 1946).

[16] A. Nadai, Theory of flow and fracture of solids, 1, p. 103 115 (McGraw-Hill Book Co., Inc., New York, N. Y., $1950)$

[17] A. E. H. Love, The mathematical theory of elasticity, 4th ed., p. 557 (Cambridge University Press, London, 1934).

Washingtor, June 1951. 